\title{
Deep Tissue Targeted Near-infrared Optogenetic Stimulation using Fully Implantable Upconverting Light Bulbs
}

\author{
Maysamreza Chamanzar, IEEE Member, David J. Garfield, Jillian Iafrati, Vikaas Sohal, Emory Chan, \\ Bruce Cohen, P. James Schuck, Michel M. Maharbiz, IEEE Senior Member
}

\begin{abstract}
We demonstrate for the first time, the possibility of targeted optogenetic stimulation of neurons deep into brain tissue $(>2 \mathrm{~mm})$ in a minimally-invasive way by sending nearinfrared light through tissue to excite passive lanthanide-doped blue-emitting upconverting nanocrystals (UCNPs) encapsulated in Parylene $\mathbf{C}$ microstructure light bulbs that emit visible (blue) light and locally excite opsins with high spatial resolution.
\end{abstract}

\section{INTRODUCTION}

Optogenetics has proven to be a powerful technique for stimulating neurons. Ion channels in specific cell-types that express light sensitive proteins (opsins) can be modulated in response to specific wavelengths of light. The absorption band of most of these opsins is in the visible range of spectrum [1], [2]. For example, the peak absorption band for Channelrhodopsins is around $475 \mathrm{~nm}$ and for Halorhodopsin is around $590 \mathrm{~nm}$. Tissue exhibits a high absorption in visible wavelength range [3]. Moreover, light is diffracted and scattered as it propagates through the tissue. The combination of large absorption and beam spreading in the tissue results in the degradation of light intensity (ratio of power to beam surface area) that rapidly falls below the excitation threshold of opsins. To compensate for this intensity drop, we need to increase the input light intensity at the surface of brain, which would cause excessive heat generation and damage to brain tissue [4]. For example, Channelrhodopsins require an average intensity of $1 \mathrm{~mW} / \mathrm{mm}^{2}$ to evoke action potentials. This means that an input power of $2.25 \mathrm{~mW}$ is required on the surface of a mouse brain from an optics fiber with a core diameter of $200 \mu \mathrm{m}$ and $\mathrm{NA}=0.37$ to excite a neuron at a depth of $2 \mathrm{~mm}$ deep in the brain tissue.

To reach deep regions of brain, fiber optics and light guides are inserted in the tissue, which can cause damage and displacement to the tissue and vasculature. Furthermore, steering the light beam in the tissue would require physically moving the guides in the tissue, causing further damage to brain tissue and vasculature.

The absorption of light in brain tissue at relevant optogenetic wavelengths is about two orders of magnitude larger than absorption of light at near-infrared (NIR) wavelengths [3]. Here, we present a novel approach to use NIR light to reach deep regions of brain and excite implanted micro light bulbs that emit visible light at the optogenetic wavelengths to locally stimulate neighboring neurons. Our light bulbs are microfabricated polymer parylene $\mathrm{C}$ pillars

*Research supported by the BayBrain Initiative.

M. Chamanzar and M. M. Maharbiz are with the University of California Berkeley, CA USA (e-mail: chamanzar@berkeley.edu).

D. Garfield, E. Chan, B. Cohen, P. J. Schuck, are with the Molecular Foundry, Lawrence Berkeley National Laboratory, CA USA.

J. Iafrati and V. Sohal are with the University of California San Francisco, CA USA. that encapsulate lanthanide-doped upconverting nanocrystals (UCNPs). These UCNPs absorb NIR and emit blue light (450nm-475nm) locally.

In this paper, we discuss the design and implementation of UCNP micro light bulbs and experimental results on thick coronal brain slices.

\section{IMPLANTABLE LIGHT BULBS}

\section{A. Upconverting Nanocrystals}

Through systematic design, the synthesis of lanthanidedoped nanocrystals is optimized [5] so that the absorbed NIR light at $\lambda=980 \mathrm{~nm}$ is upconverted efficiently to blue emission around $450 \mathrm{~nm}$, as shown in Fig. 1. Another emission band at $\lambda=800 \mathrm{~nm}$ and a weak one at $\lambda=670 \mathrm{~nm}$ are also present as a result of two photon processes in addition to three-photon processes. It was found through a combinatorial synthesis that core/shell UCNPs with core: $\mathrm{NaYF}_{4}$ alloyed with $40 \% \mathrm{Yb}, 20 \% \mathrm{Gd}$, and $2 \% \mathrm{Tm}$, shell: pure $\mathrm{NaYF}_{4}$ produced the brightest blue emission. The particles used were $12 \mathrm{~nm}$ cores surrounded by a $4 \mathrm{~nm}$ thick shell.

\section{B. Microfabrication of tetherless micro light bulbs}

To generate sufficient blue light to excite opsins and evoke action potentials, we need a large concentration of UCNPs. Also, to safely implant these small UCNPs $(\sim 20 \mathrm{~nm}$ in diameter), we need to encapsulate them in a biocompatible polymer. We chose Parylene $\mathrm{C}$ as the encapsulating medium since it is biocompatible, can be conformally deposited using a CVD method, and can be micro patterned using our previously established fabrication process [6], [7].

The fabrication process to realize micro light bulbs is outlined in Fig. 2. First, we deposit a layer of Parylene $\mathrm{C}$ to a thickness of $2 \mu \mathrm{m}$ on a bare silicon wafer. We then deposit $200 \mathrm{~nm}$ of Aluminum on top. We lithographically define the micro light bulb patterns of different sizes and wet etch Aluminum and subsequently, dry etch Parylene $\mathrm{C}$ using oxygen plasma in an ICP machine. Then we deposit a layer of photoresist as the sacrificial layer. Using an optimized etch back method, we expose the pillar tips and then etch Aluminum caps. At this step, we drop coat the UCNPs suspended in Toluene, which tend to agglomerate more on the exposed Parylene $\mathrm{C}$ regions. A capping layer of $\mathrm{SiO}_{2}$ is sputtered on top to protect UCNPs. Finally, the photoresist sacrificial layer is stripped and the pillars are released. Fig. 3 shows the confocal microscope image of an array of microfabricated encapsulating pillars with a height of $2 \mu \mathrm{m}$ and lateral dimensions of $5 \mu \mathrm{m}$. 
Figure 1. The emission spectra of lanthanide-doped UCNPs. Absorption band is centered on $\lambda=980 \mathrm{~nm}$ and the emission bands are centeered on $\lambda$ $=460 \mathrm{~nm}, \lambda=670 \mathrm{~nm}$, and $\lambda=800 \mathrm{~nm}$.



Figure 2. Fabrication process flow to realize UCNP micro light bulbs

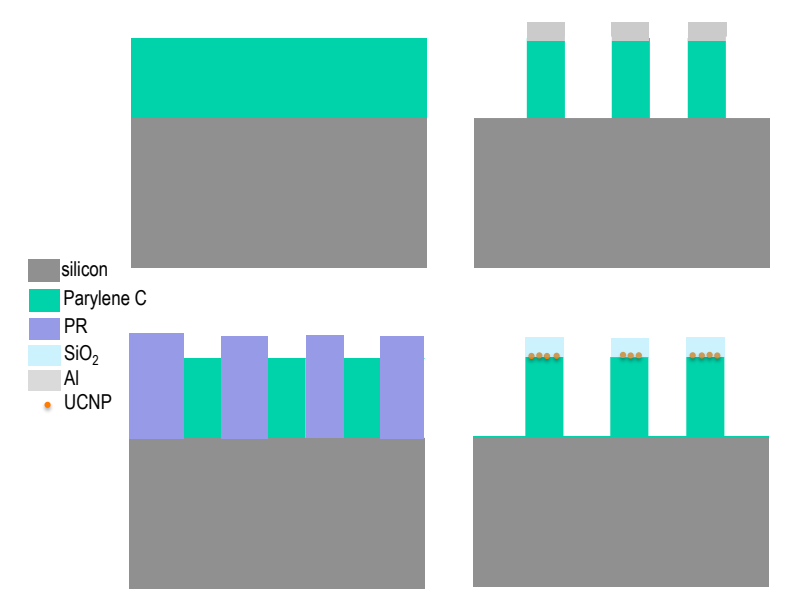

Figure 3. (a) The schematic of a realeased micro light bulb (b) Confocal microscope image of the microfabricated pillars

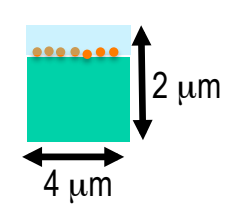

(a)

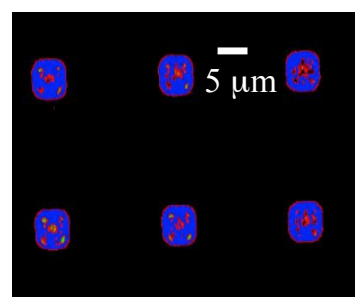

(b) $\prod_{1} 2 \mu \mathrm{m}$

\section{IMAGING THROUGH CORONAL BRAIN SLICES}

To test the possibility of exciting UCNPs embedded in parylene $\mathrm{C}$ light bulbs deep into brain tissue, we attempted to image the UCNPs through the whole thickness of sliced brain tissues of different thicknesses. We constructed sandwiched layers as shown in Fig. 4, where a layer of brain slice is sandwiched between two $250 \mu \mathrm{m}$ glass cover slips, one coated with our UCNPs. We image the emission of the UCNP light bulbs both from the top through the cover slip and also from the back through the whole thickness of the brain slice.

Animal tissue experiments were performed in accordance with protocols approved by Administrative Panels on Laboratory Animal Care at the University of California, San Francisco. Brain slices were prepared as described previously [8]. Briefly, adult C57BL/6 mice (7-10 weeks old) were anesthetized with isofluorane and decapitated. Using a vibratome (Leica VT1200 S), coronal brain slices were prepared at different thicknesses (ranging from $100 \mu \mathrm{m}$ to $3 \mathrm{~mm}$ ) in a chilled slicing solution consisting of $234 \mathrm{mM}$ sucrose, $11 \mathrm{mM}$ glucose, $24 \mathrm{mM} \mathrm{NaHCO} 3,2.5 \mathrm{mM} \mathrm{KCl}$, $1.25 \mathrm{mM} \mathrm{NaH} 2 \mathrm{PO} 4,10 \mathrm{mM} \mathrm{MgSO} 4$, and $0.5 \mathrm{mM} \mathrm{CaCl}$. Slices were then fixed overnight in $4 \%$ paraformaldehyde (Electron microscopy science) / Phosphate buffer saline $1 \mathrm{X}$ (Sigma) then mounted on glass coverslips with a glycerolbased medium (Vectashield, Vector laboratories).

Figure 4. (a) The schematic of the sample consisting of a coronal brain slice sandwitched between two glass cover slips. The top cover slip is coated with UCNP light bulbs.

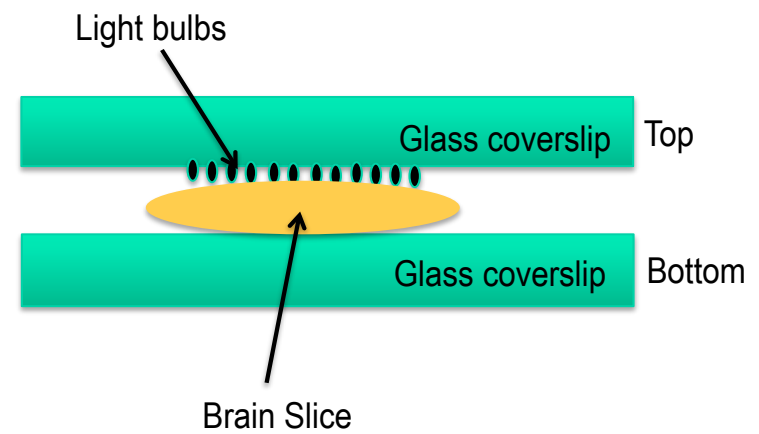

Figure 5. A brightfield microscope image of the sandwitched layer with a $2 \mathrm{~mm}$ mouse brain slice.

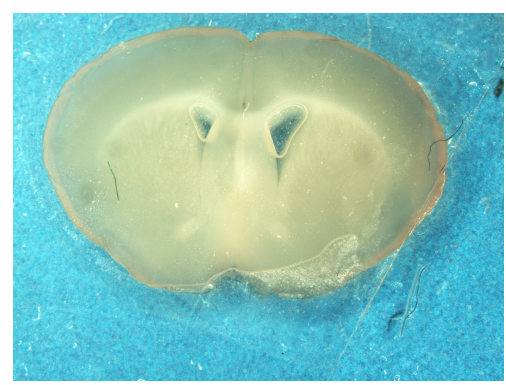


The schematic of an experimental setup to image the UCNPs is shown in Fig. 6, where an inverted microscope is used both to illuminate and image the sample. Light from a $980 \mathrm{~nm}$ laser is directed towards the sample from a 50:50 beam splitter and then focused on the sample through a $100 \mathrm{x}$ objective (NA=0.85) to a spot size of $\sim 700 \mathrm{~nm}$. The emission signal is collected through the same objective lens and beam splitter. The collected signal is shortpass filtered to remove any reflection of pump from the sample. The filtered signal is then imaged onto a CCD spectrometer.

Figure 6. Schematic of the experimental characterization setup

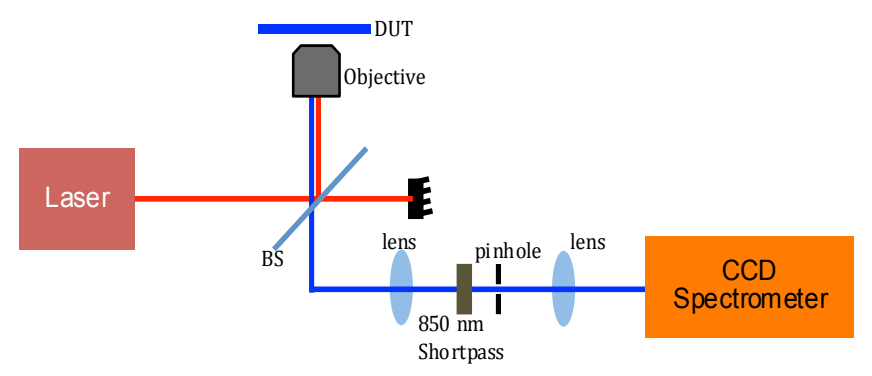

The emission signal spectrum from top of the sample is shown in Fig. 7 (obtained after $0.3 \mathrm{sec}$ integration time), where two distinct emission bands, one centered on $\lambda=800$ $\mathrm{nm}$ and the other centered on $\lambda=450 \mathrm{~nm}$ can be observed. It should be noted that the upconverting process is highly nonlinear and the emission spectra depend on the pump power. We have used a low pump intensity of $800 \mathrm{~W} / \mathrm{cm}^{2}$ to minimize damage to the brain tissue, while still being able to measure a decent emission signal level (Fig. 7). Therefore, at this low pump power, some emission bands such as the one centered on $670 \mathrm{~nm}$ cannot be picked up by the CCD spectrometer. This control experiment shows that we can image the emission of the UCNPs through the cover slip.

In the next step, we flipped the sample so that both the excitation and emission go through the whole thickness of the sample including the $2 \mathrm{~mm}$ brain slice and the cover slip. The measured spectrum is shown in Fig. 8. The existence of the $800 \mathrm{~nm}$ emission band shows that we are indeed exciting the UCNPs through the tissue and as a result blue light is emitted locally from the UCNP light bulbs. However, as expected blue light generated locally cannot propagate through the whole thickness of the brain slice and gets absorbed by the tissue. In fact, this confirms that blue light is generated locally and can excite opsins in the vicinity of UCNP light bulbs with high spatial resolution. The $800 \mathrm{~nm}$ emission band can be used to image and track particles in brain tissue. The intensity of the collected emission spectrum through the brain slice is decreased by about $86 \%$ due to scattering and absorption through the whole thickness of the brain slice tissue and the glass cover slips.

\section{OPTOGENETIC EXPERIMENTS ON LIVE CORONAL BRAIN SLICES OF A MOUSE}

As the next step to show the power of the proposed technique to reach deep regions of brain and excite opsins locally, we are performing experiments on live brain slices.
UCNP light bulbs were stereotactically injected into neocortex of 5-8 week old mice as described previously [9]. We used the heterozygous Thy1-ChR2-eYFP line 18 transgenic mice as described elsewhere [10] and also commercially available through the Jackson Laboratories [B6.Cg-Tg(Thy1-COP4/EYFP)18Gfng/J] [11]. In these mice, Channelrhodopsin-2 fused to enhanced Yellow Fluorescent protein is expressed under the control of Thy1 promoter and neuronal action potential firing happens following blue light illumination. After brain preparation from these mice, slices are incubated in artificial cerebrospinal fluid (ACSF) containing (in $\mathrm{mM}$ ): $126 \mathrm{NaCl}$, $26 \mathrm{NaHCO} 3,2.5 \mathrm{KCl}, 1.25 \mathrm{NaH} 2 \mathrm{PO} 4,1 \mathrm{MgCl} 2,2 \mathrm{CaCl}$, and 10 glucose and equilibrated with $95 \% \mathrm{O} 2$ / $5 \% \mathrm{CO} 2$.

For ex-vivo recording, slices are perfused $(2 \mathrm{ml} / \mathrm{min})$ with equilibrated ACSF at $32-35^{\circ} \mathrm{C}$. Whole-cell patch-clamp and field EPSP (fEPSP) using glass pipette electrode can be recorded from neurons at the vicinity of UCNP light bulbs. Data are collected using Multiclamp 700A and acquired with Clampex 10.2 acquisition Software via a Digidata 1440A (Axon Instruments).

Figure 7. Emission spectrum of the UCNPs from top of the sample

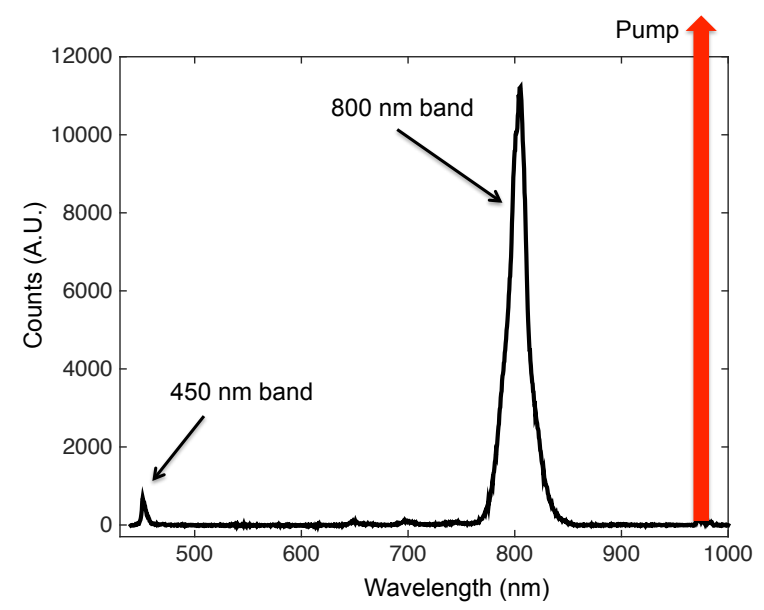

Figure 8. Emission spectrum of the UCNPs through the $2 \mathrm{~mm}$ brain slice (measured from the bottom)

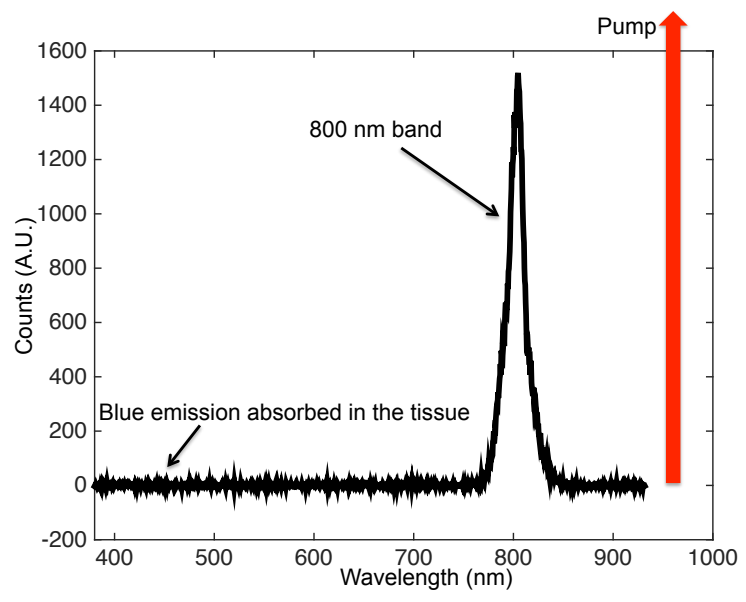


We have modified a standard upright electrophysiology rig (shown in Fig. 9) so that NIR light at $\lambda=980 \mathrm{~nm}$ can be passed through the microscope to excite the sample and visible light emission can be collected from the sample.

Evoked electrical activity can then be recorded in response to locally emitted blue light from UCNP micro light bulbs.

Figure 9. An upright electrophysiology rig, modified for NIR excitation and visible light collection and patch clamp electrophysiology recording



Further results of the optogenetic experiments on live coronal slices of transgenic will be discussed in the presentation. Our preliminary tests show that there is no evident adverse systemic effect on our transgenic mice after injection of UCNP micro light bulbs. In one case, we monitored the behavior of 4 THY 118 mice for two weeks after injection and no abnormality was observed.

\section{CONCLUSION}

Using UCNP implantable light bulbs, we can reach deep regions of brain tissue (deeper than $2 \mathrm{~mm}$ ) to stimulate opsins. We use NIR light in the optical window wavelength range $(800-1200 \mathrm{~nm})$ with minimal absorption to excite UCNP light bulbs that absorb NIR and locally emit blue light. This locally emitted blue light can stimulate opsins. Using this technique, not only can we stimulate deep regions of brain, but also we can stimulate local clusters of neurons with high spatial resolution since blue light is locally emitted in the close proximity of UCNP micro light bulbs. Beyond optogentic stimulation of neuronal activity, the proposed technique in this paper can find intriguing applications for deep tissue imaging in different biomedical applications.

\section{REFERENCES}

[1] L. Fenno, O. Yizhar, and K. Deisseroth, "The development and application of optogenetics," Annu. Rev. Neurosci., vol. 34, pp. 389-412, 2011.

[2] E. S. Boyden, "A history of optogenetics: the development of tools for controlling brain circuits with light," F1000 Biol. Rep., vol. 3, 2011

[3] S. L. Jacques, "Optical properties of biological tissues: a review.," Phys. Med. Biol., vol. 58, no. 11, pp. R37-61, Jun. 2013.

[4] L. Yang and S. J. Miklavcic, "Revised Kubelka-Munk theory. III. A general theory of light propagation in scattering and absorptive media," J. Opt. Soc. Am. A, vol. 22, no. 9, p. 1866, 2005.

[5] D. J. Gargas, E. M. Chan, A. D. Ostrowski, S. Aloni, M. V. P. Altoe, E. S. Barnard, B. Sanii, J. J. Urban, D. J. Milliron, B. E. Cohen, and P. J. Schuck, "Engineering bright sub-10-nm upconverting nanocrystals for single-molecule imaging.," Nat. Nanotechnol., vol. 9, no. April, Mar. 2014.
[6] P. Ledochowitsch, E. Olivero, T. Blanche, and M. M. Maharbiz "A transparent $\mu \mathrm{ECoG}$ array for simultaneous recording and optogenetic stimulation.," Conf. Proc. IEEE Eng. Med. Biol. Soc., vol. 2011, pp. 2937-40, Jan. 2011.

[7] R. Muller, H.-P. Le, W. Li, P. Ledochowitsch, S. Gambini, T. Bjorninen, A. Koralek, J. M. Carmena, M. M. Maharbiz, E. Alon, and J. M. Rabaey, "24.1 A miniaturized 64-channel $225 \mu \mathrm{W}$ wireless electrocorticographic neural sensor," in 2014 IEEE International Solid-State Circuits Conference Digest of Technical Papers (ISSCC), 2014, pp. 412-413.

[8] S. Gee, I. Ellwood, T. Patel, F. Luongo, K. Deisseroth, and V. S. Sohal, "Synaptic activity unmasks dopamine D2 receptor modulation of a specific class of layer $\mathrm{V}$ pyramidal neurons in prefrontal cortex.," J. Neurosci., vol. 32, no. 14, pp. 4959-71, Apr. 2012.

[9] V. S. Sohal, F. Zhang, O. Yizhar, and K. Deisseroth, "Parvalbumin neurons and gamma rhythms enhance cortical circuit performance.," Nature, vol. 459, no. 7247, pp. 698-702, Jun. 2009.

[10] B. R. Arenkiel, J. Peca, I. G. Davison, C. Feliciano, K. Deisseroth, G. J. Augustine, M. D. Ehlers, and G. Feng, "In vivo lightinduced activation of neural circuitry in transgenic mice expressing channelrhodopsin-2.," Neuron, vol. 54, no. 2, pp. 205 18, Apr. 2007.

[11] "jaxmice.jax.org." 